\title{
Study of Cu-doping effects on magnetic properties of Fe-doped ZnO by first principle calculations
}

\author{
A EL AMIRI*, H LASSRI ${ }^{\dagger}, M$ ABID and E K HLIL ${ }^{\dagger+}$ \\ Laboratoire de Physique Fondamentale et Appliquée (LPFA), Faculté des Sciences Ain Chock, \\ Université Hassan II, B.P. 5366 Mâarif, Casablanca, Morroco \\ ${ }^{\dagger}$ Laboratoire de Physique des Matériaux, Micro-électronique, Automatique et Thermique (LPMMAT), \\ Faculté des Sciences Ain Chock, Université Hassan II, B.P. 5366 Mâarif, Casablanca, Morrocco \\ ${ }^{+\dagger}$ Institut Néel, CNRS - Université J. Fourier, B.P. 166, 38042 Grenoble, France
}

MS received 16 May 2013; revised 7 September 2013

\begin{abstract}
Using ab initio calculations on $\mathrm{Zn}_{0.975-x} \mathrm{Fe}_{0.025} \mathrm{Cu}_{x} \mathrm{O}(x=0,0.01,0.02,0.05)$, we study the variations of magnetic moments vs $\mathrm{Cu}$ concentration. The electronic structure is calculated by using the KorringaKohn-Rostoker (KKR) method combined with coherent potential approximation (CPA). We show that the total magnetic moment and magnetic moment of Fe increase on increasing $\mathrm{Cu}$ content. From the density of state (DOS) analysis, we show that $\mathrm{Cu}$-induced impurity bands can assure, by two mechanisms, the enhancement of Fe magnetic moment in $\mathrm{Zn}_{0.975-x} \mathrm{Fe}_{0.025} \mathrm{Cu}_{x} \mathrm{O}$.
\end{abstract}

Keywords. (Fe, Cu)-doped ZnO; diluted magnetic semiconductors; DOS.

\section{Introduction}

Magnetism and semiconducting properties can coexist in semiconductor materials by introducing a small fraction of magnetic impurity atoms such as $\mathrm{Mn}, \mathrm{Cr}, \mathrm{Co}, \mathrm{Ni}, \mathrm{Fe}$ and $\mathrm{Cu}$. This category of semiconductors is called diluted magnetic semiconductors (DMSs). Diluted magnetic semiconductors are expected to play an important role in materials science and electronics. In these materials in addition to the charge, the spin degrees of freedom also play an important role in the physical properties. From IIVI compound semiconductors, $\mathrm{ZnO}$ has attracted great attention. It has a wide direct bandgap of $3 \cdot 3 \mathrm{eV}$ (LandoltBörnstein 2002). ZnO doped with $3 d$ transition metal (TM) elements have been studied widely since the calculation model by Dietl et al (2008) who have predicted the possibility of ferromagnetism in $\mathrm{ZnO}$ with a small amount of Mn as an impurity. Several magnetic, magnetooptical and magneto-transport properties were observed in $\mathrm{ZnO}$ systems doped by transition metals, including Mn-added ZnO (Fukumura et al 1999; Ando et al 2001; Jin et al 2001; Wi et al 2004; Piamba et al 2011; Iqbal et al 2011), Fe-added ZnO (Ueda et al 2001; Mok Cho et al 2002; Piamba et al 2011), Co-added ZnO (Ueda et al 2001; Lee et al 2002; Mok Cho et al 2002; Prellier et al 2003; Venkatesan et al 2004; Wi et al 2004) and Ni-added ZnO (Lee et al 2002; Prellier et al 2003; Ekicibil et al 2012).

*Author for correspondence (aelamiri@casablanca.ma)
Moreover, using first-principle calculations, Sato and Katayama-Yoshida (2000) have demonstrated theoretically that a $\mathrm{ZnO}$ matrix doped with $\mathrm{TM}$ atoms such as $\mathrm{V}$, $\mathrm{Cr}, \mathrm{Fe}, \mathrm{Co}$ and Ni exhibited ferromagnetic ordering. Furthermore, many $a b$ initio calculations were performed on two metals co-doped $\mathrm{ZnO}$. For example, He et al (2008) showed the origin of ferromagnetism in ( $\mathrm{Ga}, \mathrm{Co})$-doped $\mathrm{ZnO}$ doped El Amiri et al (2012) explained the enhancement of total and Co magnetic moments in (Co, Cu)doped $\mathrm{ZnO}$.

Recently, many researchers tried to dope other ions into $\mathrm{ZnO}$ matrix to obtain two metal ions co-doped $\mathrm{ZnO}$ in order to enhance its optical and magnetic properties by introducing additional carriers (Chakraborti et al 2007; Li et al 2007; Xu et al 2008; Brihi et al 2010; Dinesha et al 2010). Lin et al (2004) supposed that a small amount of additional $\mathrm{Cu}$-doping should create additional carriers in Co-doped $\mathrm{ZnO}$, and its magnetization would be greatly enhanced in bulk samples. In this work, we have performed $a b$ initio calculations on $\mathrm{Zn}_{0 \cdot 975-x} \mathrm{Fe}_{0 \cdot 025} \mathrm{Cu}_{x} \mathrm{O}$. We show that the total moment and magnetic moment per atom of iron increase by increasing $\mathrm{Cu}$ concentration.

\section{Electronic and magnetic structure calculations}

Electronic structure calculations were performed using the Korringa-Kohn-Rostoker (KKR) method within the density functional theory (Akai 1989). To take into account the random distribution of $\mathrm{Fe}$ and $\mathrm{Cu}$ in $\mathrm{Zn}$ sites, we used the coherent potential approximation (CPA). Vosko, Wilk 
and Nusair (VWN) parametrization of the exchangecorrelation energy functional was used (Vosko et al 1980). The form of crystal potential is approximated by a muffin-tin potential and the wavefunctions in the respective muffin-tin spheres were expanded in real harmonics up to $l=2$, where $l$ is the quantum angular momentum defined at each site. Spin polarization, relativistic effect and spin-orbit interaction were taken into account. $\mathrm{Zn}_{0.975-x} \mathrm{Fe}_{0.025} \mathrm{Cu}_{x} \mathrm{O}$ is considered to crystallize in wurtzite structure and we assume that $\mathrm{Fe}$ and $\mathrm{Cu}$-doping do not affect the experimental lattice constants much ( $a=3.25 \AA$ and $c=5 \cdot 21 \AA$ ) for $\mathrm{ZnO}$ (Kisi and Elcombe 1989).

\section{Results and discussion}

We focus on the magnetic moments of $\mathrm{Zn}_{0.975-x}$ $\mathrm{Fe}_{0.025} \mathrm{Cu}_{x} \mathrm{O},(x=0,0 \cdot 02,0 \cdot 05)$. Using $a b$ initio calculations, we found that both the total magnetic moment and magnetic moment of Fe increase by increasing $\mathrm{Cu}$ concentration. Figure 1 presents variation of the total moment and Fe moment per atom. These results show that the increase of $\mathrm{Cu}$-doping concentration from 0 to 0.05 , leads to an increase of both the total moment and iron moment from 0.22 to $0.27 \mu_{\mathrm{B}}$ and from 3.47 to $3.61 \mu_{\mathrm{B}}$, respectively. Recently, Zhang et al (2007) have shown that saturation magnetizations $\left(M_{\mathrm{s}}\right)$ are $1.8 \mu_{\mathrm{B}} / \mathrm{Fe}^{2+}$ and $2.7 \mu_{\mathrm{B}} / \mathrm{Fe}^{2+}$ for $\mathrm{Zn}_{0.975} \mathrm{Fe}_{0.025} \mathrm{O}$ and $\mathrm{Zn}_{0.97} \mathrm{Fe}_{0.025} \mathrm{Cu}_{0.005} \mathrm{O}$, respectively. In order to explain this trend of the magnetic moment, we analyse the electron density of states (DOS). First, the valence-electron configurations of $\mathrm{Zn}$ and substituting $\mathrm{Fe}$ and $\mathrm{Cu}$ atoms are $3 d^{10} 4 s^{2}, 3 d^{6} 4 s^{2}$ and $3 d^{10} 4 s^{1}$, respectively. According to the first hand's rule, the minimum of the total energy of an isolated $3 d$ atom corresponds to the occupation of the $3 d$ orbitals that gives the highest spin moment. Therefore, the minority $3 d$ states of Fe must be partially occupied whereas those of $\mathrm{Cu}$ are almost fully occupied. Indeed, the calculated

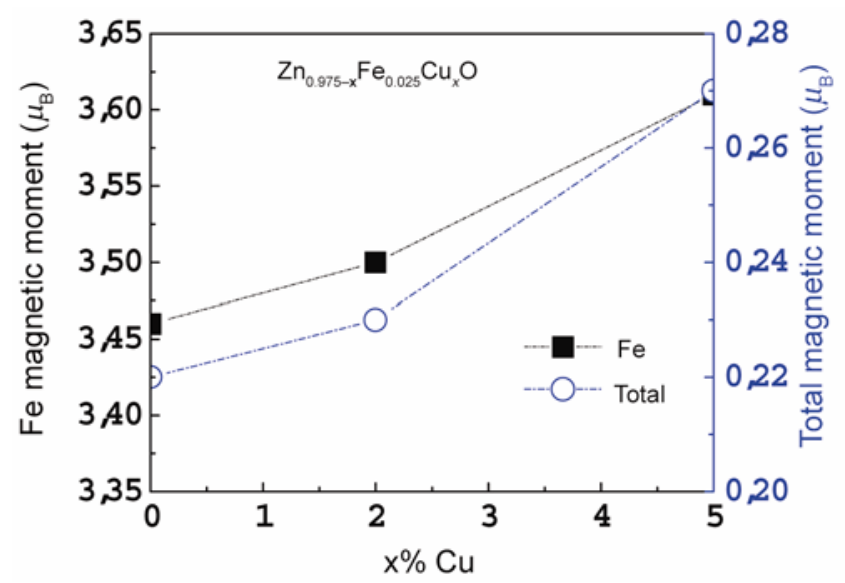

Figure 1. Variation of total and $\mathrm{Fe}$ magnetic moments in $\mathrm{Zn}_{0.975-x} \mathrm{Fe}_{0.025} \mathrm{Cu}_{x} \mathrm{O}(x=0,0 \cdot 02,0 \cdot 05)$ samples. electronic structures of $3 d$-states of $\mathrm{Zn}, \mathrm{Fe}$ and $\mathrm{Cu}$ in $\mathrm{Zn}_{0.975-x} \mathrm{Fe}_{0.025} \mathrm{Cu}_{x} \mathrm{O}$ DOS are in agreement with this assumption (see figure 2(a and b)). Secondly, in figure 3, the total DOS of $\mathrm{Zn}_{0.975-x} \mathrm{Fe}_{0.025} \mathrm{Cu}_{x} \mathrm{O}(x=0,0.02,0.05)$ are reported. In particular, in figure 3(a), the region where DOS is zero presents the bandgap of the host matrix with a value almost equal to $2 \mathrm{eV}$. Moreover, in figure $3(\mathrm{a}-\mathrm{c})$, we note that the region between 0 and $-1 \cdot 36 \mathrm{eV}$ opens as $\mathrm{Cu}$ concentration increases, indicating that the local density of states nearing to left of the Fermi level becomes slightly important. This can be explained by the creation of shallow donor levels caused by $\mathrm{Cu}$ introduction. Indeed, as observed in figures 2(a and b), these levels are mainly originating from $\mathrm{Cu}-3 d$ bands. Moreover, $\mathrm{Cu}-3 d$ bands lie exactly in such region $(-1 \cdot 36-0 \mathrm{eV})$ existing in the bandgap of $\mathrm{Zn}_{0.975} \mathrm{Fe}_{0.025} \mathrm{O}$ DOS (see figure 3(a)), so Cu-introduction creates donor impurity bands. According to our band structure calculations, we can observe the overlap between $\mathrm{Fe}-3 d$ and $\mathrm{Cu}-$ induced impurity band states for the spin-down around the Fermi level (figure 2(a and b)) which leads to significant DOS at Fermi level and contributes to the enhancement of the total moment. This overlap also leads to
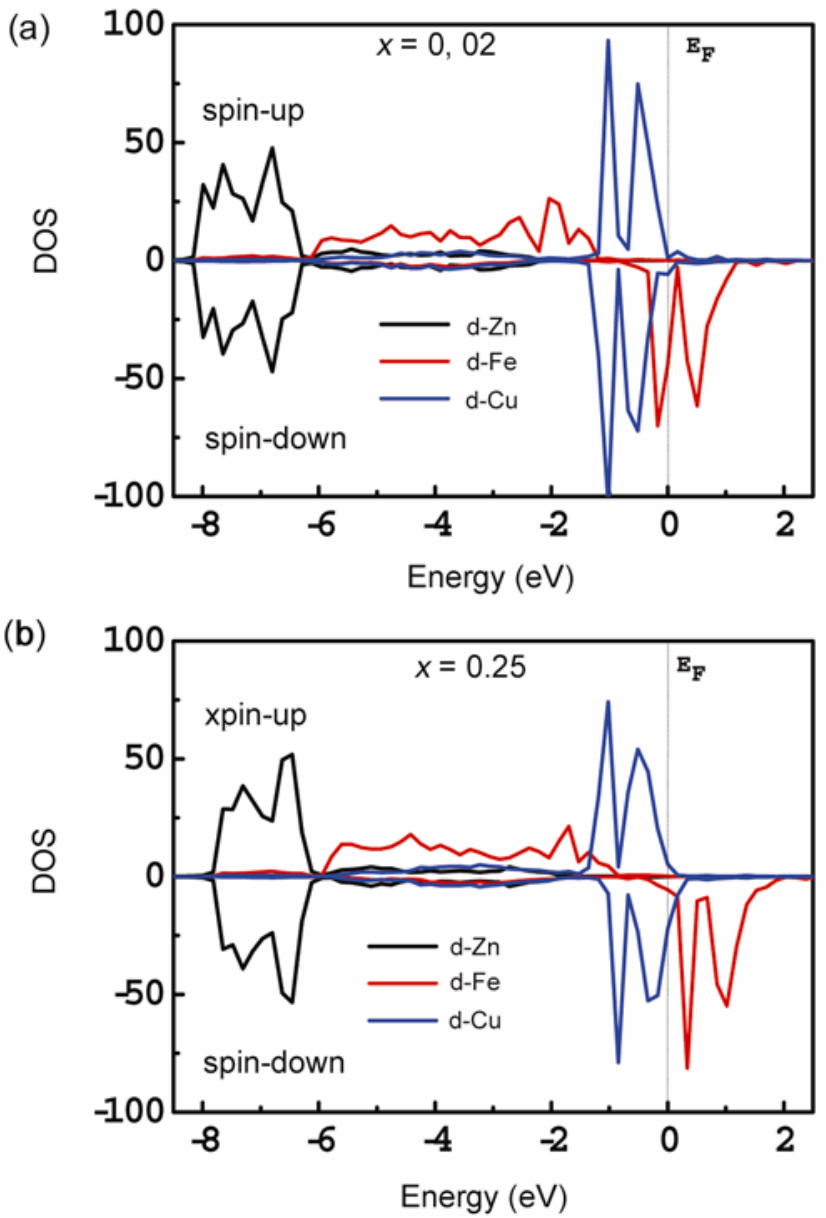

Figure 2. (a-b) Partial DOS of $\mathrm{Zn}, \mathrm{Fe}$ and $\mathrm{Cu}$ in $\mathrm{Zn}_{0 \cdot 975-x}$ $\mathrm{Fe}_{0.025} \mathrm{Cu}_{x} \mathrm{O}(x=0.02,0.05)$ samples. 
hybridization between the involved spin-down states. Such hybridization facilitates mutual charge transfer between $\mathrm{Cu}$ and $\mathrm{Fe}$ atoms which favours interaction between the localized moments of Fe atoms. This interaction tends to align Fe moments and contributes to increase the charge polarization (spin-up-spin-down) for $\mathrm{Fe}$ atoms which explains the results listed in table 1 and consequently the increase of magnetic moment of iron. So, we

(a)

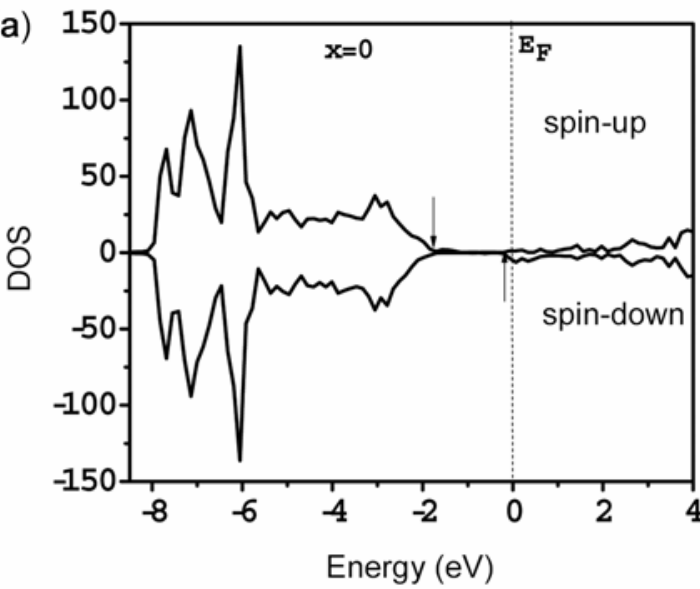

(b)

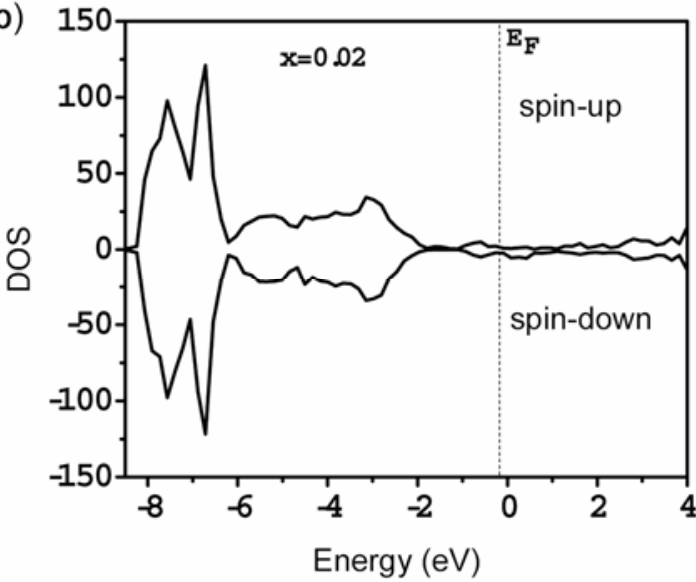

(c)

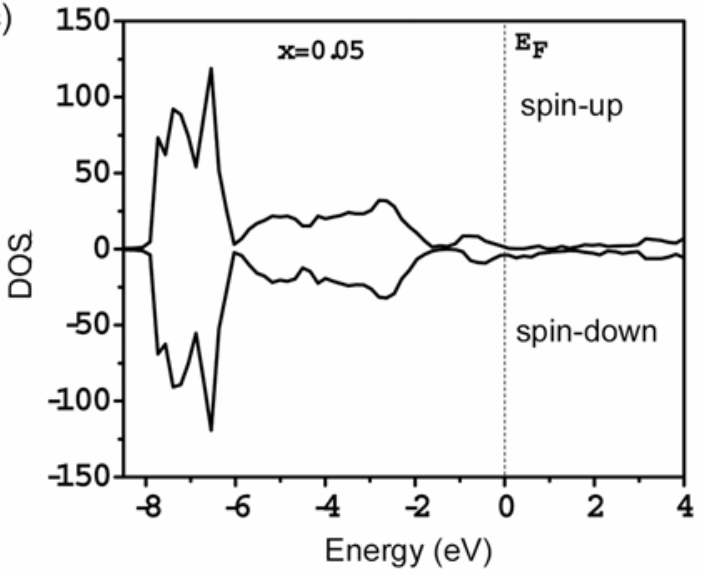

Figure 3. (a-c) Total DOS of $\mathrm{Zn}_{0 \cdot 975-x} \mathrm{Fe}_{0.025} \mathrm{Cu}_{x} \mathrm{O} \quad(x=0$, $0 \cdot 02,0 \cdot 05)$ samples. can conclude that there is a superexchange interaction between $\mathrm{Fe}$ atoms caused by $\mathrm{Cu}$-induced donor impurity bands. This interaction is expected to enhance the ferromagnetic ordering between Fe atoms. This assumption is also confirmed by our calculations listed in table 2, where we have reported the difference between the energies of antiferromagnetic (AFM) and ferromagnetic (FM) configurations $\Delta E=E_{\mathrm{AFM}}-E_{\mathrm{FM}}$. Positive value of $\Delta E$ means that $\mathrm{Fe}$ atoms prefer FM spin order. Also the exchange parameter $J$ (see table 2) was derived from $\Delta E$ by using the following equation.

$$
\frac{\Delta E}{2}=-J S(S+1)
$$

where $S$ is the Fe magnetic moment and $J$ the exchange parameters of exchange between Fe atoms. We find that without $\mathrm{Cu}$ and $\mathrm{Fe}$, atoms choose the AFM spin-order. Soumahoro et al (2010) reported that the anti-ferromagnetic contribution increases with iron concentration in Fe-doped $\mathrm{ZnO}$ films. As seen in table 2, we note also, that $\Delta E$ and $J$ become positive and increase with $\mathrm{Cu}$ concentration. So $\mathrm{Cu}$ incorporation in Fe-doped $\mathrm{ZnO}$ enforce the ferromagnetic coupling between Fe atoms. This can be explained by the fact that the copper atoms are logged between $\mathrm{Fe}$ atoms and consequently favours super exchange interaction between Fe atoms. Working on similar components, Fengchun et al (2011) reported that activation of the ferromagnetism of (Co, Cu)-doped $\mathrm{ZnO}$ relative to Co-doped $\mathrm{ZnO}$ is due to the effective hybridization between Co-3d states and the $\mathrm{Cu}$-induced donor impurity band at the Fermi level which is consistent with our results. Another possible explanation of the enhancement of magnetic moment of iron is the role of donor impurity bands on the magnetic couplings. Recently, Coey et al (2005) proposed that the ferromagnetic exchange couplings mediated by shallow donor electrons that formed bound magnetic polarons (BMP) in oxide DMSs (Venkatesan et al 2004; Coey et al 2005). In our work, the $\mathrm{Cu}$ cation substitution for $\mathrm{Zn}^{2+}$ ions introduces easily shallow donor levels in the bandgap and therefore, contributes to BMP formation which favours a

Table 1. Valence charge of Fe.

\begin{tabular}{cccc}
\hline & Up & Down & Up/Down \\
\hline 0 & 4,33 & 0,86 & 3,47 \\
2 & 4,34 & 0,84 & 3,5 \\
5 & 4,38 & 0,77 & 3,61 \\
\hline
\end{tabular}

Table 2. Variation of $\Delta E$ and $J$ vs content of $\mathrm{Cu}$.

\begin{tabular}{lcc}
\hline$x(\% \mathrm{Cu})$ & $E_{\mathrm{AFM}}-E_{\mathrm{FM}}(\mathrm{meV})$ & $J(\mathrm{meV})$ \\
\hline 0 & -0.3 & $-9.52 \times 10^{-3}$ \\
2 & 1.35 & $4.28 \times 10^{-2}$ \\
5 & $5 \cdot 3$ & 0.16 \\
\hline
\end{tabular}


ferromagnetic exchange coupling between $\mathrm{Fe}$ ions and consequently increase of $\mathrm{Fe}$ magnetic moment. Both interpretations are well confirmed by our DOS calculations, so we can conclude that both mechanisms can contribute to the enhancement of ferromagnetism in $\mathrm{Cu}-\mathrm{Co}$ doped $\mathrm{ZnO}$.

\section{Conclusions}

In this work, we have performed $a b$ initio calculations on $\mathrm{Zn}_{0.975-x} \mathrm{Fe}_{0.025} \mathrm{Cu}_{x} \mathrm{O}(x=0,0.01,0.02,0.05)$. We have reported that the magnetic moment per $\mathrm{Fe}$ atom increases with increasing $\mathrm{Cu}$ content. Also, using total and partial DOS, we are able to show that $\mathrm{Cu}$-induced impurity bands ensure by two mechanisms, the enhancement of Fe magnetic moment in $\mathrm{Zn}_{0.975-x} \mathrm{Fe}_{0.025} \mathrm{Cu}_{x} \mathrm{O}$ systems.

\section{References}

Akai H 1989 J. Phys. Condens. Matter 18045

Ando K, Saito H, Jin Z, Fukumura T, Kawasaki M, Matsumoto $\mathrm{Y}$ and Koinuma H 2001 J. Appl. Phys. 897284

Brihi N, Bouaine A, Berbadj A, Schmerber G, Colis S and Dinia A 2010 Thin Solid Films 5184549

Chakraborti D, Ramachandran S, Trichy G and Narayan J 2007 J. Appl. Phys. 101053918

Coey J M D, Venkatesan M and Fitzgerald C B 2005 Nat. Mater. 4173

Dietl T, Ohno H, Matsukura F, Cibert J and Ferrand D 2000 Science 2871019

Dinesha M L, Jayanna H S, Mohanty S and Ravi S $2010 \mathrm{~J}$. Alloys Compd. 490618

Ekicibil A, Bulun G, Kilic S, Cetin, Dikmen Z and Orhun O 2012 J. Supercond. Nov. Magn. 25435
El Amiri A, Abid M, Lassri H and Hlil E K 2012 J. Supercond. Nov. Magn. 252529

Fengchun Hu et al 2011 J. Appl. Phys. 109103705

Fukumura T, Jin Z, Ohtomo A, Koinuma H and Kawasaki M 1999 Appl. Phys. Lett. 753366

He Y et al 2008 Phys. Rev. B78 155202

Iqbal Javed, Liu Xiaofang, Majid Abdul and Yu Ronghai 2011 J. Supercond. Nov. Magn. 24699

Jin Z, Fukumura T, Kawasaki M, Ando K, Saito H and Sekiguchi T 2001 Appl. Phys. Lett. 783824

Kisi E H and Elcombe M M 1989 Acta Crysttallogr. 451867

Landolt-Börnstein 2002 Condensed matter New Series, Group III (Heidelberg: Springer-Verlag)

Lee H J, Jeong S-Y, Ryong Cho C and Hong Park C 2002 Appl. Phys. Lett. 814020

Li G R, Qu D L, Zhao W X and Tong Y X 2007 Electrochem. Commun. 91661

Lin H T, Chin T S, Shih J C, Lin S H and Hong T M 2004 Appl. Phys. Lett. 85621

Mok Cho Y, KilChoo W, Kim H, Kim D and Eon Ihm Y 2002 Appl. Phys. Lett. 803358

Piamba J F, Paz J C, Ligia E Zamora and Perez Alcazar G A 2011 J. Supercond. Nov. Magn. 252223

Prellier W, Fouchet A, Mercey B, Simon Ch and Raveau B 2003 Appl. Phys. Lett. 823490

Sato K and Katayama-Yoshida H 2000 Jpn J. Appl. Phys. 39 L555

Soumahoro I et al 2010 Thin Solid Films 5184593

Ueda K, Tabata H and Kawai T 2001 Appl. Phys. Lett. 79 988

Venkatesan M, Fitzgerald C B, Lunney J G and Coey J M D 2004 Phys. Rev. Lett. 93177206

Vosko S H, Wilket L and Nusair M 1980 Can. J. Phys. 581200

Wi S C et al 2004 Appl. Phys. Lett. 844233

Xu Q et al 2008 Thin Solid Films 5161160

Zhang Hua-Wei, Wei Zhi-Ren, Li Zhi-Qiang and Dong Guo-Yi 2007 Mater. Lett. 613605 